\title{
Development and Comparative Analysis of Geospatial Feature Automatic Extraction System in Open-source Environment
}

\author{
Dong Gook Lee, ${ }^{1}$ Ji Ho You, ${ }^{2 *}$ and Hyun Jik Lee ${ }^{3 * *}$ \\ ${ }^{1}$ ALLforLAND Co., Ltd., 145, Gasan digital 1-ro, Geumcheon-gu, Seoul 08506, Republic of Korea \\ ${ }^{2}$ Chung-ang Aerosurvey Co., Ltd., 146-1, Tongil-ro, Jongno-gu, Seoul 03180, Republic of Korea \\ ${ }^{3}$ Department of Civil Engineering, Sangji University, \\ 83 Sangjidae-gil, Wonju-si, Gangwon-do 26339, Republic of Korea \\ (Received February 24, 2021; accepted May 14, 2021)
}

Keywords: open source, geospatial feature extraction system, development, classification accuracy

The aim of this study is to develop a system for geospatial feature extraction from images to be obtained from CAS500-1/2 satellites currently being developed by the Ministry of Land, Infrastructure, and Transport, Republic of Korea. The feasibility of automatic geospatial feature extraction is verified by applying the relative radiometric normalization technique to KOMPSAT-3A satellite images, which are expected to have similar specifications to CAS500 images. Furthermore, the developed system is compared with commercial software to verify its classification accuracy. In this study, two KOMPSAT-3A satellite images were collected and relative radiometric normalization was performed. Identical parameters and threshold values were applied to both the commercial software and the developed system to extract geospatial features by feature class and analyze the classification accuracy using an error matrix. Image segmentation and image classification were performed for grassland, ground, roads, buildings, and water bodies. The results indicated a classification accuracy of $90 \%$ or higher, which was set as the accuracy goal. The difference in the classification accuracy of the two systems was less than approximately $1 \%$, implying comparable performances for the two systems. Using the geospatial feature extraction system developed by us, it is expected that basic data will be generated for monitoring national territory such as forests and urban areas.

\section{Introduction}

The Ministry of Land, Infrastructure, and Transport (MOLIT), Republic of Korea, is currently developing CAS500-1/2 satellites that can capture images with a ground sample distance of $0.5 \mathrm{~m}$. These satellites are to be used for land observation and monitoring. Meanwhile, land use classification and geospatial feature extraction technology, change detection and time-series monitoring technology, and digital surface model (DSM) and digital terrain model (DTM) extraction technology are currently under development for the purposes of monitoring land. ${ }^{(1)}$ In particular, land use classification and geospatial feature extraction can provide excellent indicators of the environment ${ }^{(2,3)}$ and environmental changes. ${ }^{(4)}$ For land use

${ }^{*}$ Corresponding author: e-mail: sjce96@daum.net

** Corresponding author: e-mail: hjiklee@sangji.ac.kr

https://doi.org/10.18494/SAM.2021.3344 
classification and geospatial feature extraction from high-resolution satellite images, an objectbased classification method, which classifies a group of pixels recognized together as an object, is commonly used. This method has been proven effective for high-resolution satellite images such as those from ASTER, ${ }^{(5)}$ KOMPSAT-2, ${ }^{(6)}$ QuickBird, ${ }^{(7-9)}$ and WorldView-3. ${ }^{(10)}$ Pixel-based classification is another method used for land use classification and geospatial feature extraction. However, pixel-based classification techniques are not as efficient as object-based classification techniques for high-resolution satellite images. ${ }^{(11)}$ Examples of object-based classification that detects objects through segmentation and classification of images include agricultural land extraction, ${ }^{(12,13)}$ urban tree extraction, ${ }^{(14)}$ and land cover classification. ${ }^{(15-18)}$

Typical remote sensing technologies that use satellite images include change detection technology, along with geospatial feature extraction techniques. ${ }^{(19,20)}$ Factors affecting the results of change detection include the geometrical relationship of the sun-target sensor, resolution differences between sensors, and differences in atmospheric and ecological conditions. ${ }^{(21)}$ Therefore, preprocessing is required to obtain consistent change detection results. Images related to spectral values are preprocessed using absolute or relative radiometric normalization methods. ${ }^{(22)}$ Absolute radiometric normalization applies accurate atmospheric input data and the spectral profile of images to a mathematical model and converts a digital number to a scaled surface reflectance. However, actual atmospheric observation data are difficult to collect, and this method cannot be used if there is no ground observation value with respect to the acquisition time. In contrast, relative radiometric normalization can be used to normalize the spectral characteristics of multiperiod images based on the spectral characteristics of the reference image. It can be used in different locations because it does not require various weather data or models and performs normalization by estimating the relative change rates in multiperiod images. ${ }^{(23-27)}$

In this study, we hypothesize that automation-oriented object-based geospatial feature extraction will be feasible if an object-based classification method is applied to high-resolution multiperiod satellite images following relative radiometric normalization. We collected KOMPSAT-3A satellite images, which are expected to have similar specifications to CAS500$1 / 2$ satellite images. In addition, we performed relative radiometric normalization and objectbased geospatial feature extraction using a reference image and a relative radiometric normalized image. We executed an automation-oriented object-based geospatial feature extraction process that applies parameters and a threshold range to both images identically during the image segmentation and classification stage, and we analyzed whether the extracted geospatial features had similar shapes. Furthermore, we attempted to develop a system capable of performing geospatial feature extraction based on open-source software (SW). To this end, we selected SAGA GIS, which is open-source SW, as a development platform and used various spatial analysis libraries to implement the geospatial feature extraction process. The developed geospatial feature extraction system and commercial SW were used to extract geospatial features, and the performance of the proposed system was verified through a comparative analysis. Our newly developed system for spatial object extraction is expected to be available for use even by non-experts of remote exploration. Figure 1 shows an overview of the study. 


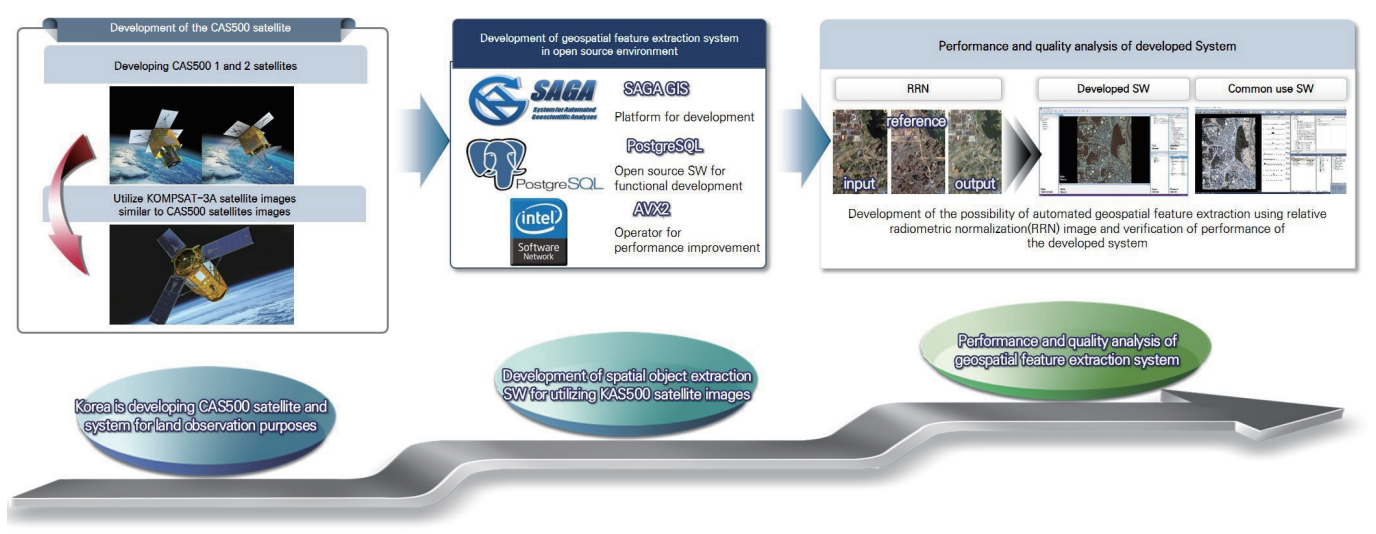

Fig. 1. (Color online) Study overview.

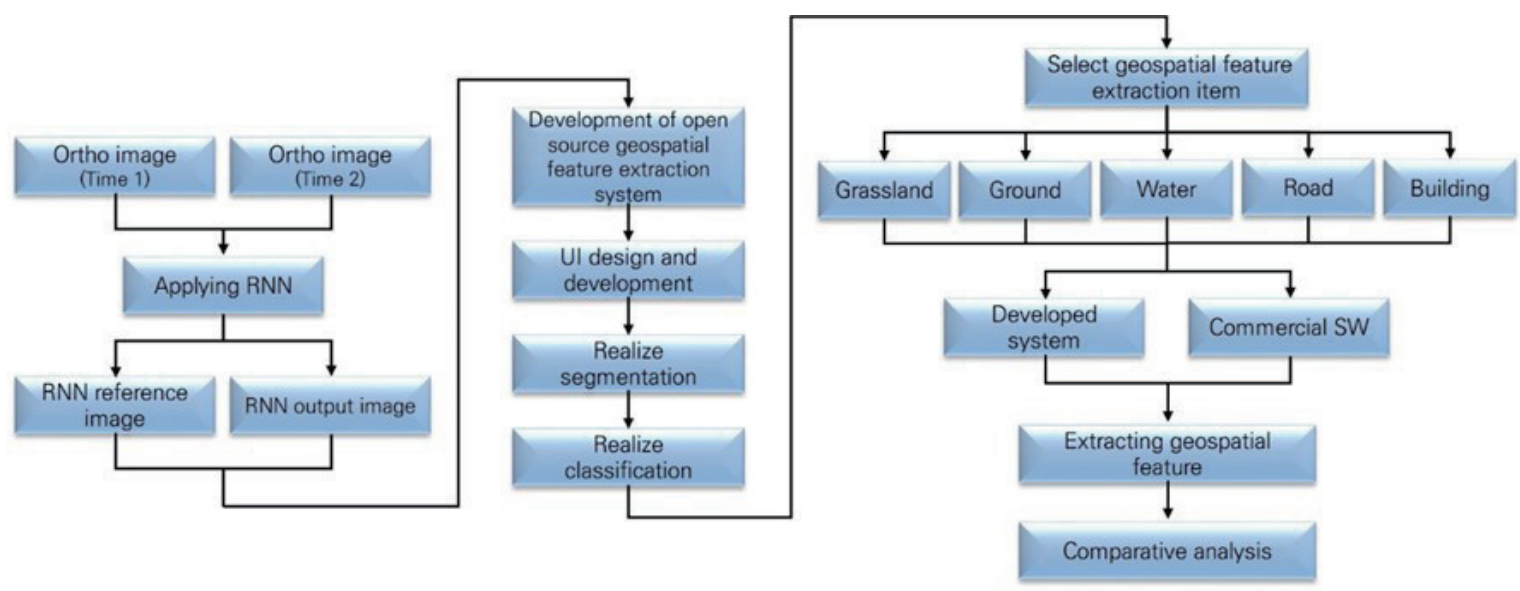

Fig. 2. (Color online) Flowchart of this study.

\section{Materials and Methods}

In Republic of Korea, CAS500-1/2 satellites are currently under development, along with the related technologies and SW for geospatial feature extraction, relative radiometric normalization, change detection, and DSM/DTM extraction of the images captured by the satellites. In this study, relative radiometric normalization technology, a preprocessing technology for change detection and time-series monitoring that is currently under development, is applied to the proposed system in an open-source environment for automation-oriented object-based geospatial feature extraction. Furthermore, the performance of the developed system is verified by comparing it with that of commercial SW. Figure 2 shows a flowchart of this study.

\subsection{Study area}

An area corresponding to Jijeong-ri, Baeksan-myeon, Gimje-si, Jeollabuk Province, Republic of Korea, was selected as the study area (Fig. 3). The top left point of the area is located at 


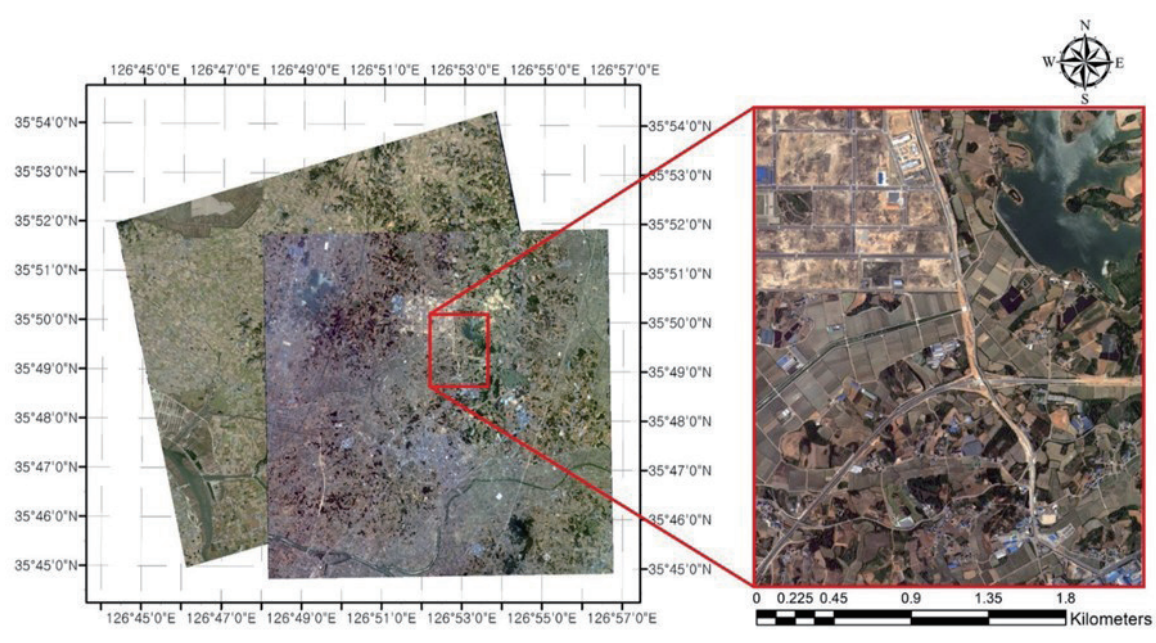

Fig. 3. (Color online) Study area.

$35^{\circ} 50^{\prime} 59.9972$ ", $126^{\circ} 52^{\prime} 29.2939^{\prime \prime}$ and the bottom right point is located at $35^{\circ} 49^{\prime} 30.0033^{\prime \prime}$, $126^{\circ} 54^{\prime} 0.6695^{\prime}$, with the elevation ranging from approximately 5 to $30 \mathrm{~m}$.

\subsection{Satellite images used for study}

Because the CAS500-1/2 satellites are currently under development, it is impossible to obtain images from these satellites. Therefore, this study was performed using KOMPSAT-3A satellite images, which are expected to have similar specifications to CAS500-1/2 satellite images. The KOMPSAT-3A satellite images collected for the study comprised multispectral data captured in June 2015 and October 2017 and pansharpened true orthoimages of the satellite (L2G) composed of red (R), green (G), blue (B), and near-infrared (NIR) in the GeoTIFF format (Fig. 4).

The KOMPSAT-3A satellite images used in this study were purchased from Korea Aerospace Research Institute through the Arirang Satellite Image Search and Order System portal. Table 1 shows the specifications of the KOMPSAT-3A satellite images.

\section{Development of Geospatial Feature Extraction System in Open-source Environment}

\subsection{Design of geospatial feature extraction system}

As shown in Fig. 5, geospatial feature extraction methods are classified into serial and parallel processes. The former produces a thematic map, while the latter extracts specific geospatial features to meet the given purpose. The serial process extracts objects sequentially in order of decreasing size. Therefore, it requires a long time to extract objects, especially small objects, and objects that the user does not need are also extracted. In contrast, the parallel process uses specific objects as spatial information. Unlike the serial process, it only extracts specific objects and is expected to facilitate automation. ${ }^{(28)}$ In this study, we used the open- 


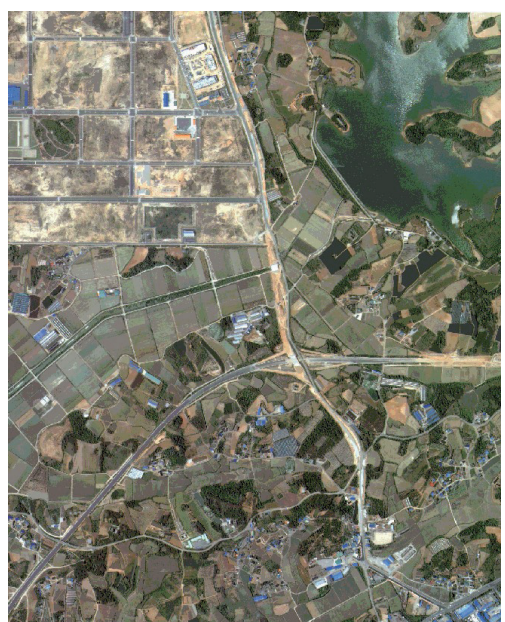

(a)

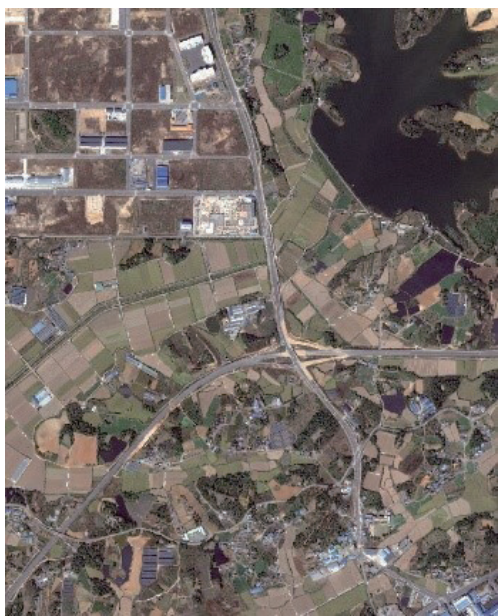

(b)

Fig. 4. (Color online) Orthoimages of KOMPSAT-3A satellite collected for this study: (a) satellite image captured in June 2015; (b) satellite image captured in October 2017.

Table 1

Specifications of the satellite images used in this study.

\begin{tabular}{|c|c|c|c|c|c|}
\hline \multirow{2}{*}{ Satellite } & \multirow{2}{*}{ Acquisition data } & \multirow{2}{*}{$\begin{array}{l}\text { Ground sample } \\
\text { distance }\end{array}$} & \multirow{2}{*}{ Cloud level } & \multicolumn{2}{|c|}{ Tilt angle } \\
\hline & & & & Roll & Pitch \\
\hline \multirow{2}{*}{ KOMPSAT-3A } & 2015.06 .06 & Approx. $0.55 \mathrm{~m}$ & A & $-7.7432020^{\circ}$ & $-0.2370128^{\circ}$ \\
\hline & 2017.10 .21 & Approx. $0.55 \mathrm{~m}$ & A & $12.9447022^{\circ}$ & $-18.6244520^{\circ}$ \\
\hline \multicolumn{6}{|c|}{ KOMPSAT-3A satellite specifications } \\
\hline \multicolumn{2}{|c|}{ Spectral bands } & \multicolumn{2}{|c|}{ Optics } & Data quantization & Swath width \\
\hline PAN & $450-900 \mu \mathrm{m}$ & \multirow{5}{*}{$\begin{array}{l}\text { Focal length } \\
\text { F number }\end{array}$} & \multirow{5}{*}{$\begin{array}{l}8.6 \mathrm{~m} \\
\mathrm{f} / 11.5\end{array}$} & \multirow{5}{*}{14 bit } & \multirow{5}{*}{$\begin{array}{l}12 \mathrm{~km} \\
\text { (at nadir) }\end{array}$} \\
\hline Blue & $450-520 \mu \mathrm{m}$ & & & & \\
\hline Green & $520-600 \mu \mathrm{m}$ & & & & \\
\hline Red & $630-690 \mu \mathrm{m}$ & & & & \\
\hline NIR & $760-900 \mu \mathrm{m}$ & & & & \\
\hline
\end{tabular}

source SW SAGA GIS as the development platform to design a system that enables parallelprocess-based geospatial feature extraction to facilitate automation.

\subsubsection{Implementation of image segmentation algorithm}

The object-based classification technique applied for geospatial feature extraction must perform image segmentation. Therefore, we implemented a seed region growing algorithm used in SAGA GIS. This algorithm extracts a seed and forms a cluster from the extracted seed using the standard deviation and mean of the surrounding pixels.

Seed extraction is performed as shown in Fig. 6. An input image is first downsampled and then restored using the B-spline curve. In a search area of $3 \times 3$ pixels that are normalized relative to the variance, if the variance value of the center pixel is greater than that of the surrounding pixels, it is selected as a seed. 

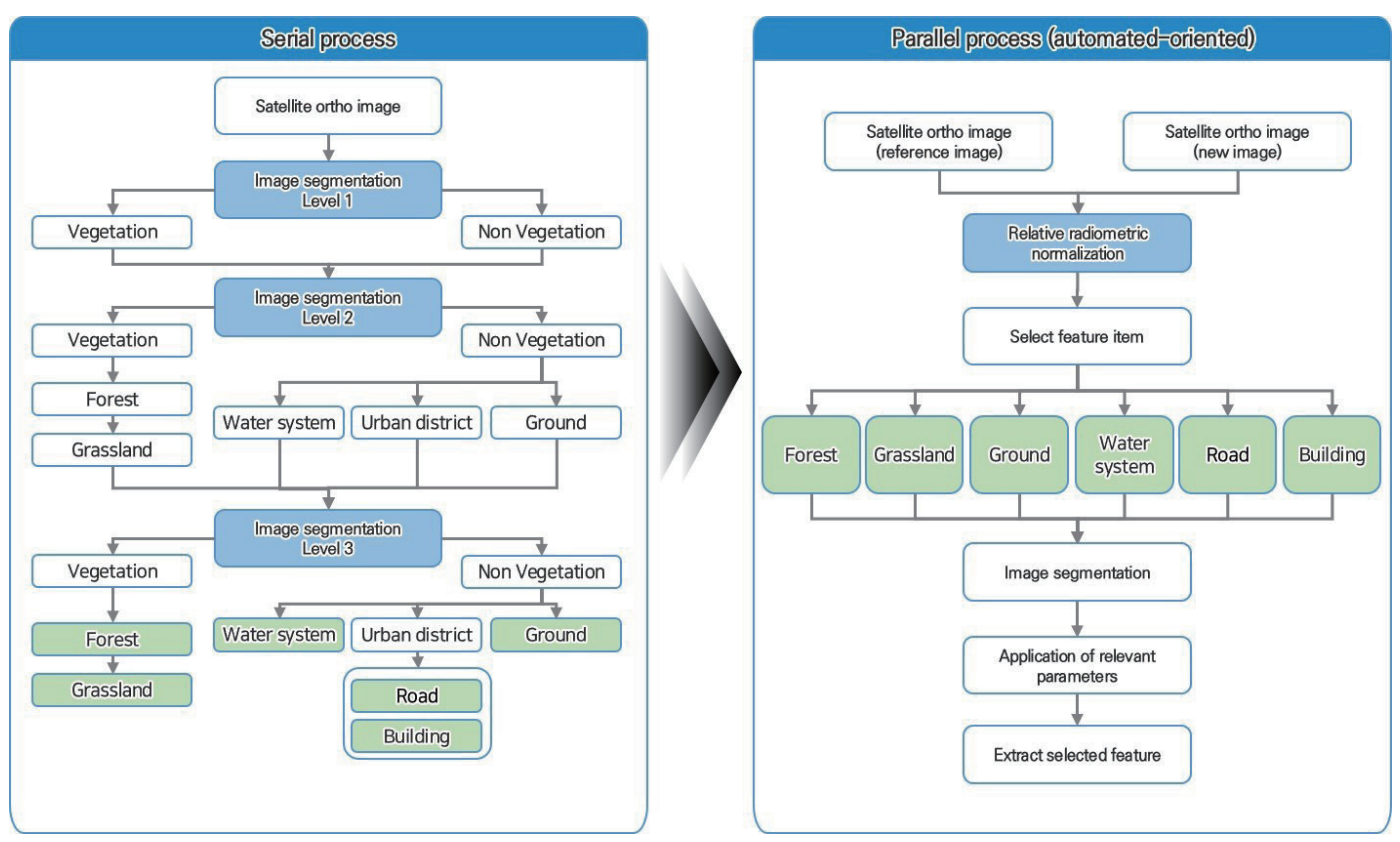

Fig. 5. (Color online) Comparison of geospatial feature extraction processes.

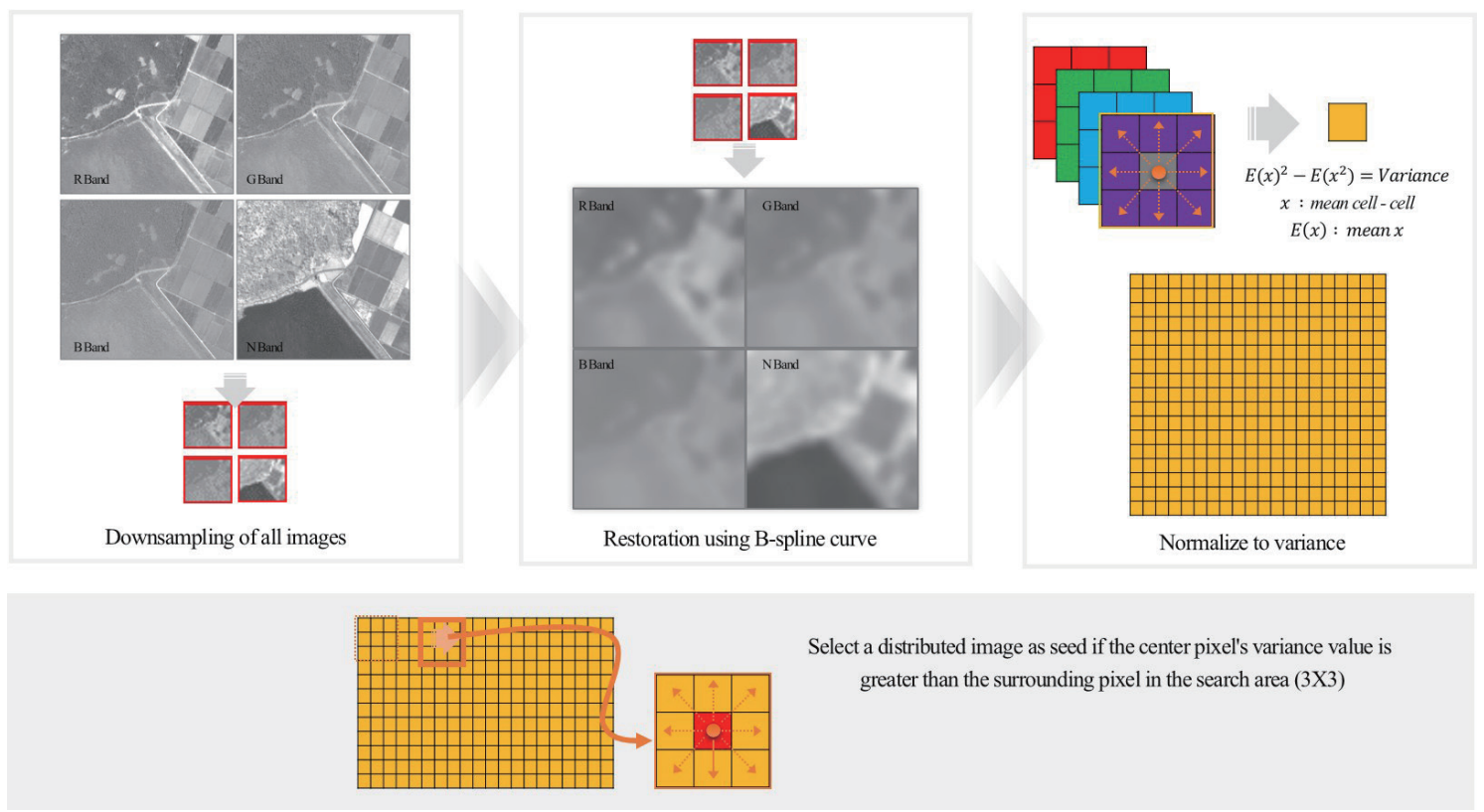

Fig. 6. (Color online) Seed extraction method for image segmentation.

A search based on the extracted seed is performed along eight surrounding directions, and the standard deviation and mean are calculated relative to cluster pixels with high similarity, thereby segmenting the image, as shown in Fig. 7.

When a pixel cluster is generated, error pixels not belonging to the pixel cluster can also be included. To remove such error pixels, surrounding pixels are searched, and majority filtering is performed to create high-frequency pixel clusters, as shown in Fig. 8. Image segmentation is 

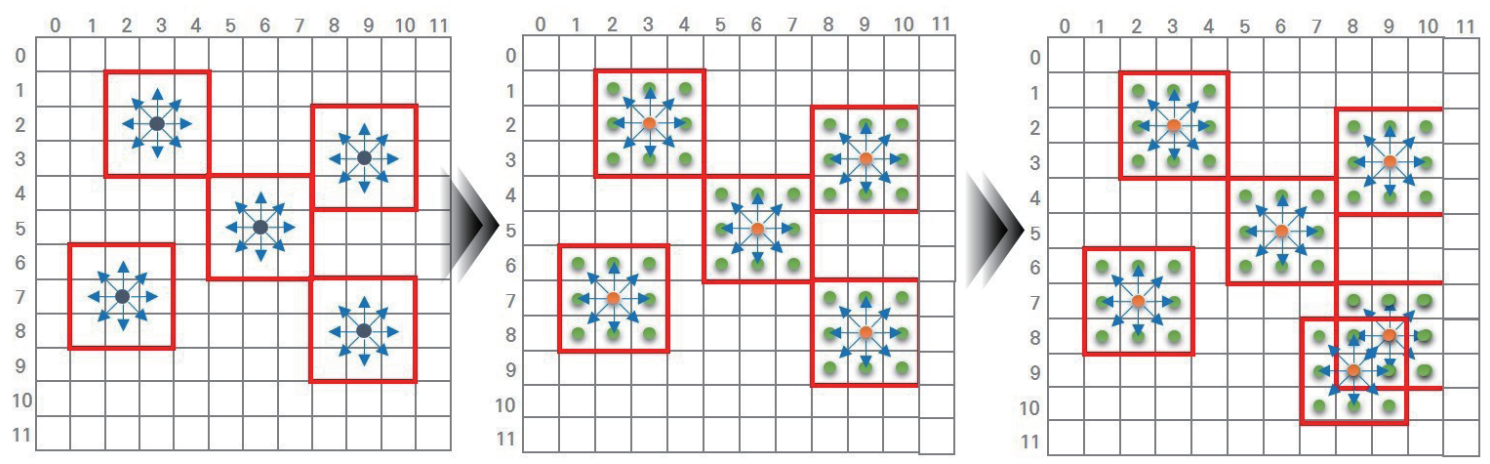

Fig. 7. (Color online) Process of generating pixel clusters using seeds.
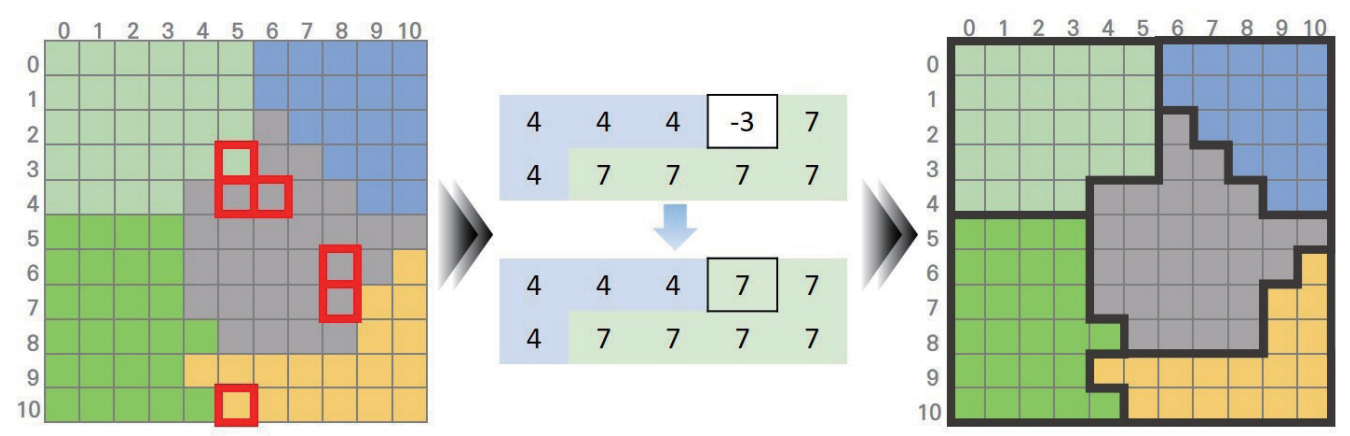

Fig. 8. (Color online) Error pixel correction method based on majority filtering.

completed by generating a cluster of corrected pixels, and the data are generated on the basis of the pixel values corresponding to the vertices of the pixel cluster.

\subsubsection{Implementation of classification function}

When image segmentation is performed, clusters containing pixels with high similarity are generated. To perform image classification, these clusters should contain parameters. Accordingly, clusters of pixels with high similarity are generated in the shape of a polygon, and for each cluster polygon, the spectrum average, standard deviation, and spectrum ratio calculated from the pixels of the image by band are input as attributes. In addition, spectral indices such as the normalized difference vegetation index (NDVI) and normalized difference water index (NDWI) are input as attributes. These attributes are used as parameters for image classification and geospatial feature extraction (Table 2).

\subsection{High-speed geospatial feature extraction}

The segmentation of a satellite image of $9041 \times 10771$ pixels using the seed region growing algorithm in the SAGA GIS required more than $3 \mathrm{~h}$, which can be inconvenient from a user perspective. To speed up the data processing for geospatial feature extraction, we used Intel's Advanced Vector Extensions 2 (AVX2), which serializes two-dimensional image data into one- 
Table 2

Parameters for image classification and geospatial feature extraction.

\begin{tabular}{llcc}
\hline Parameter & Equation & Parameter & Equation \\
$\begin{array}{l}\text { Spectrum } \\
\text { average }\end{array}$ & $\frac{\sum_{i=1}^{n L} \mu_{i}}{n_{L}}$ & Spectrum ratio & $\frac{\mu_{L}}{\sum_{i=1}^{n L} \mu_{i}}$ \\
\hline NDVI & $\frac{N i r-R e d}{N i r+R e d}$ & $\begin{array}{c}\text { Standard } \\
\text { deviation }\end{array}$ & $\sqrt{\frac{1}{\# P_{v}}\left(\sum_{(x, y, z, t) \in P_{v}} c_{k}^{2}(x, y, z, t)-\frac{1}{\# P_{v}}\left(\sum_{(x, y, z, t) \in P_{v}} c_{k}(x, y, z, t)\right)^{2}\right)}$ \\
\hline NDWI & $\frac{G r e e n-N i r}{\text { nDSM }}$ & Slope & $\tan ($ slopeaverage $)=\sqrt{\left(\frac{\delta Z}{\delta X}\right)^{2}+\left(\frac{\delta Z}{\delta Y}\right)^{2}}$ \\
\hline
\end{tabular}
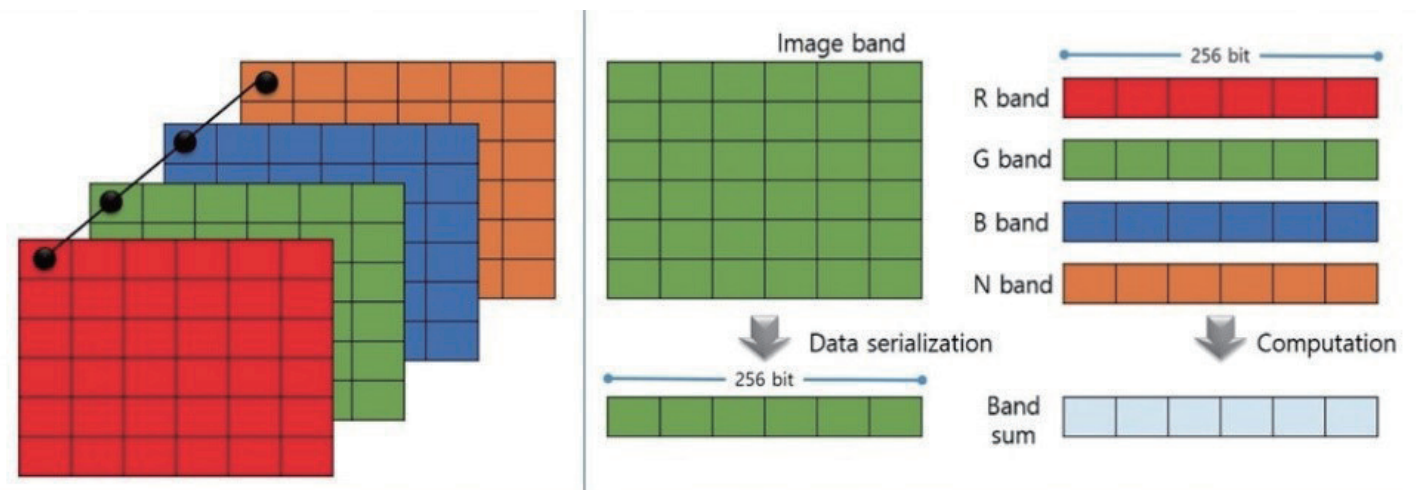

\begin{tabular}{|l|l|l|l|l|l|l|l|l|l|l|l|l|l|l|l|l|l|l}
\hline \hline Image1 & 100 & 110 & 110 & 110 & 100 & 110 & 100 & 105 & 100 & 105 & 105 & 105 & 95 & 100 & 95 & 95 & & $\ldots . .$. \\
\hline Image2 & 100 & 100 & 110 & 110 & 100 & 110 & 100 & 105 & 100 & 105 & 105 & 105 & 100 & 100 & 95 & 95 & & $\ldots$. \\
\hline
\end{tabular}

\section{\begin{tabular}{|l|l|l|l|l|l|l|l|l|l|l|l|l|l|l|l|l|}
\hline Result & 200 & 210 & 220 & 220 & 200 & 220 & 200 & 210 & 200 & 210 & 210 & 210 & 195 & 200 & 190 & 190 \\
\hline
\end{tabular}}

Fig. 9. (Color online) AVX2 and OpenMP processing for high-speed image data processing.

dimensional data and enables faster data processing via parallel processing based on open multiprocessing (OpenMP), as shown in Fig. 9. This reduces the image loading time to approximately $1 \mathrm{~s}$. Furthermore, the segmentation process only required approximately $20 \mathrm{~min}$, although this varied depending on the scale factor setting.

\section{Geospatial Feature Extraction Using Commercial SW and Proposed System Based on Open-source SW}

\subsection{Extraction of relative radiometric normalization}

Differences between images of heterogeneous periods can be attributed to various causes such as weather conditions, atmospheric effects, and sensor characteristics at the time of image 
capture. However, when performing relative radiometric normalization, the difference in brightness between images can be minimized by normalizing the brightness value between the images from heterogeneous periods.

For automation-oriented object-based geospatial feature extraction, there must be no difference between the brightness values for different images of the same area. However, two images captured at different times cannot match precisely for various reasons, such as weather and atmospheric effects. Therefore, in this study, we performed relative radiometric normalization to minimize the difference in brightness values between multiperiod images and applied the same parameters and threshold values to the multiperiod images during object-based geospatial feature extraction.

For relative radiometric normalization, the scattergram between the NIR bands of the nochange region of the input image and reference image is used to extract the no-change regions. After selecting the spectrum indices, such as NDVI and NDWI, as parameters, multilayerperceptron-based ecological normalization is performed. Relative radiometric normalization is then performed using histogram-matching-based postprocessing ${ }^{(29)}$ (Fig. 10).

\subsection{Parameters and thresholds for geospatial feature extraction}

Geospatial features are extracted through a classification process after image segmentation by using the set parameters and thresholds. The parameters and thresholds derived for grassland, ground, roads, buildings, and water bodies are shown in Fig. 11.

\subsection{Accuracy goal set for geospatial feature extraction and accuracy analysis}

Studies related to land use classification and geospatial feature extraction were reviewed, and it was found that the classification accuracy goal of extracted geospatial features was
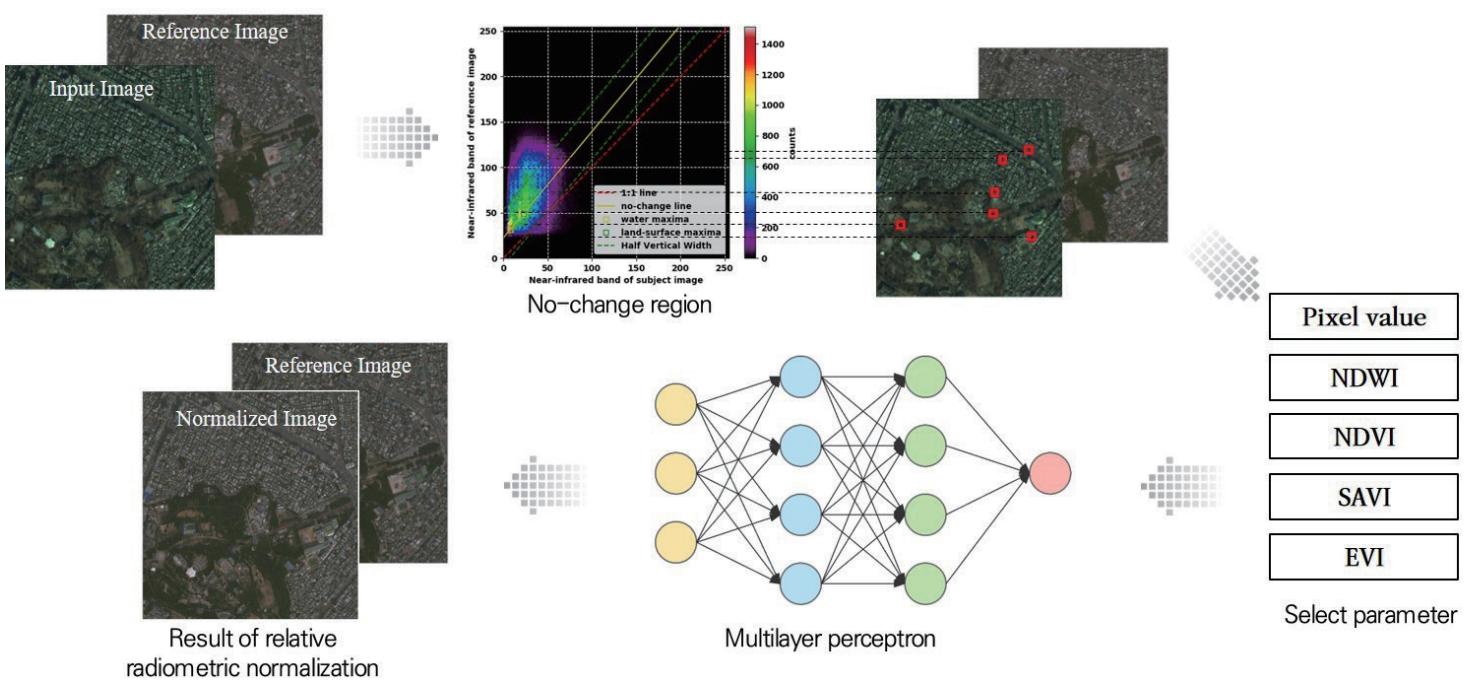

Fig. 10. (Color online) Schematic of the relative radiometric normalization algorithm applied in this study. 


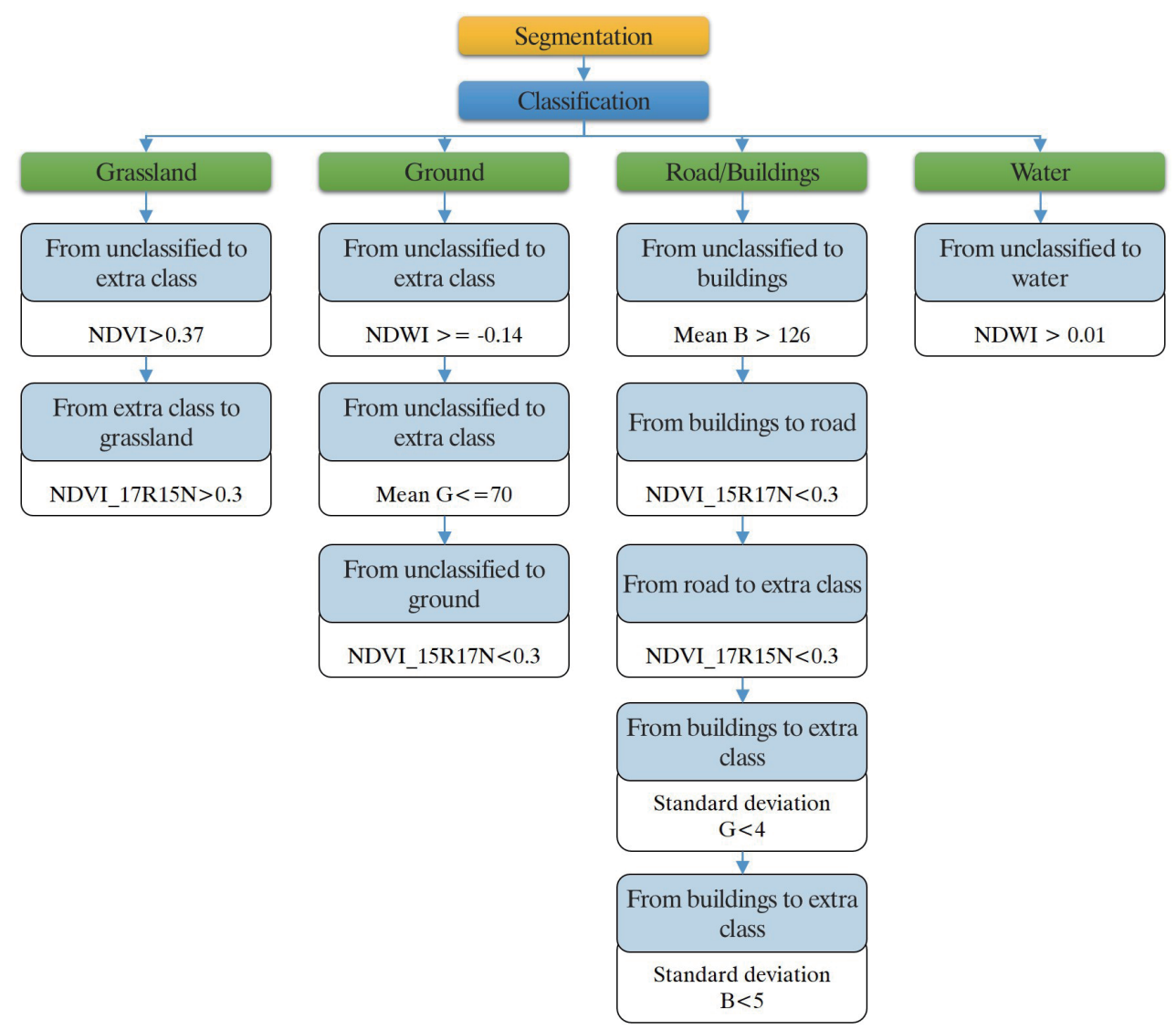

Fig. 11. (Color online) Parameters and thresholds for geospatial feature extraction applied in this study.

$70-90 \% .^{(30-32)}$ Therefore, the accuracy goal was set to $90 \%$ or higher in this study. In general, classification accuracy analysis is based on calculations using an error matrix consisting of $k$ classes. In this study, since geospatial features were extracted by feature class, the classification accuracy analysis was performed using a $2 \times 2$ matrix, as shown in Fig. 12 .

\section{Results}

\subsection{Result of relative radiometric normalization}

As shown in Fig. 13, the image from June 2015 was set as the reference image, and relative radiometric normalization was performed on the image from October 2017. The geospatial features were extracted by applying identical parameters and threshold values to both the relative radiometric normalized image and the reference image.

\subsection{Results of geospatial feature extraction using commercial SW and developed system}

The user interface (UI) of the geospatial feature extraction system was designed to show the process of extracting spatial features using satellite images and to provide various attribute 


\begin{tabular}{|c|c|c|c|c|}
\hline & \multicolumn{3}{|c|}{ Ground Reference Test Information } & \multirow[b]{2}{*}{ Row total } \\
\hline \multirow{3}{*}{$\begin{array}{c}\text { Remote } \\
\text { Sensing } \\
\text { Classification }\end{array}$} & Class & $\begin{array}{c}\text { Classified } \\
\text { feature }\end{array}$ & $\begin{array}{c}\text { Unclassified } \\
\text { feature }\end{array}$ & \\
\hline & $\begin{array}{l}\text { Classified } \\
\text { feature }\end{array}$ & $x_{1,1}$ & $x_{1,2}$ & $x_{1+}$ \\
\hline & $\begin{array}{c}\text { Unclassified } \\
\text { feature }\end{array}$ & $x_{2,1}$ & $x_{2,2}$ & $x_{+2}$ \\
\hline & Column total & $x_{+1}$ & $x_{+2}$ & $\mathrm{~N}$ \\
\hline
\end{tabular}

Fig. 12. (Color online) Error matrix for classification accuracy analysis.
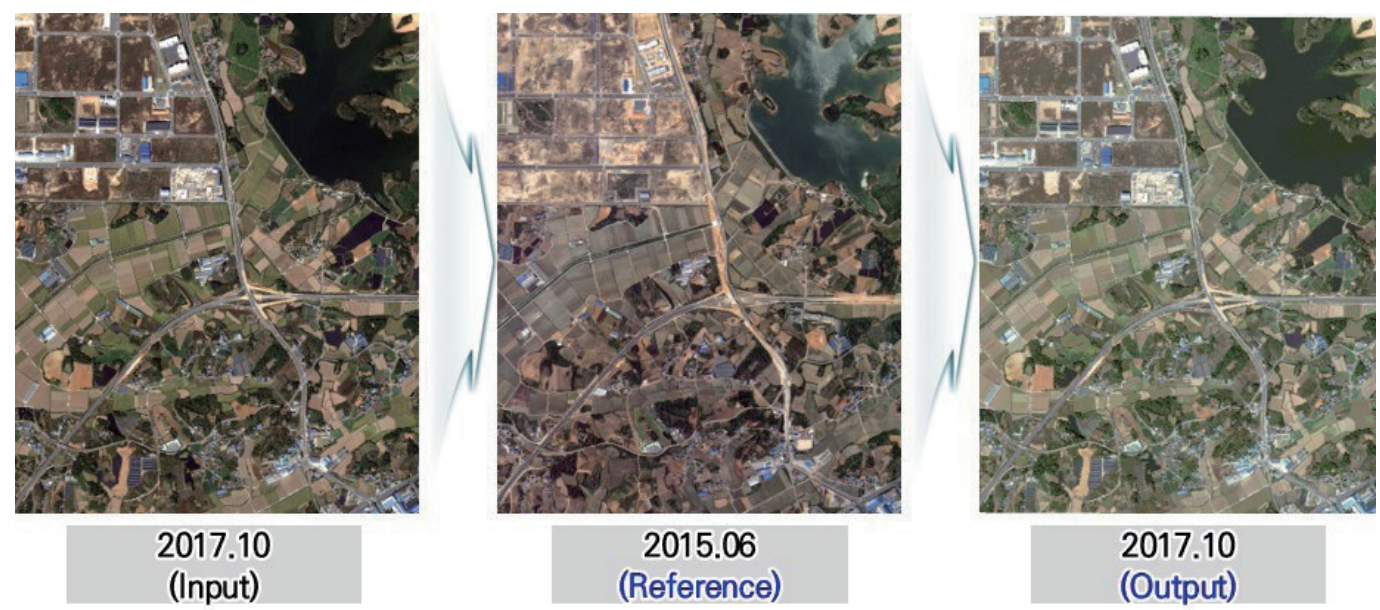

Fig. 13. (Color online) Results of relative radiometric normalization.

properties and layers of information. The proposed geospatial feature extraction system was developed in an open-source environment, as shown in Fig. 14.

The parameters and threshold values derived in this study were applied identically to both the reference image and the relative radiometric normalized image in the developed system and commercial SW; thereby grassland, ground, roads, buildings, and water bodies were extracted.

\subsubsection{Geospatial feature extraction results for reference image}

The reference image was used as the standard for relative radiometric normalization. The image captured in June 2015 was set as the reference image. Figures 15 and 16 show the geospatial features extracted using the commercial SW and the developed system, respectively. The extracted geospatial features demonstrate similar performances of both systems.

\subsubsection{Geospatial feature extraction results for relative radiometric normalized image}

Relative radiometric normalization was performed on the image captured in October 2017 to produce an image with similar brightness values to the reference image. Subsequently, geospatial features were extracted by feature class from the relative radiometric normalized image by 


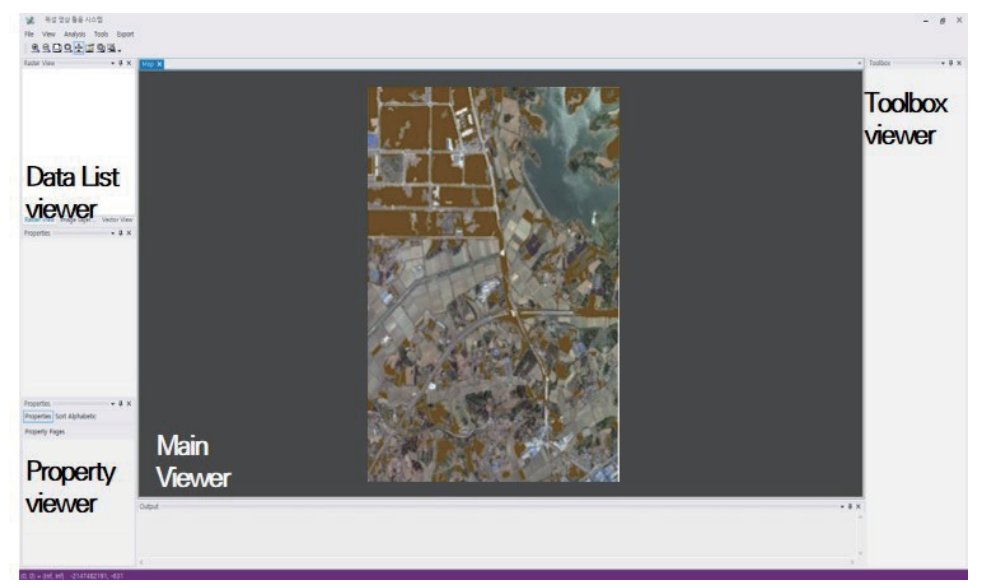

Fig. 14. (Color online) Geospatial feature extraction system developed in open-source environment.
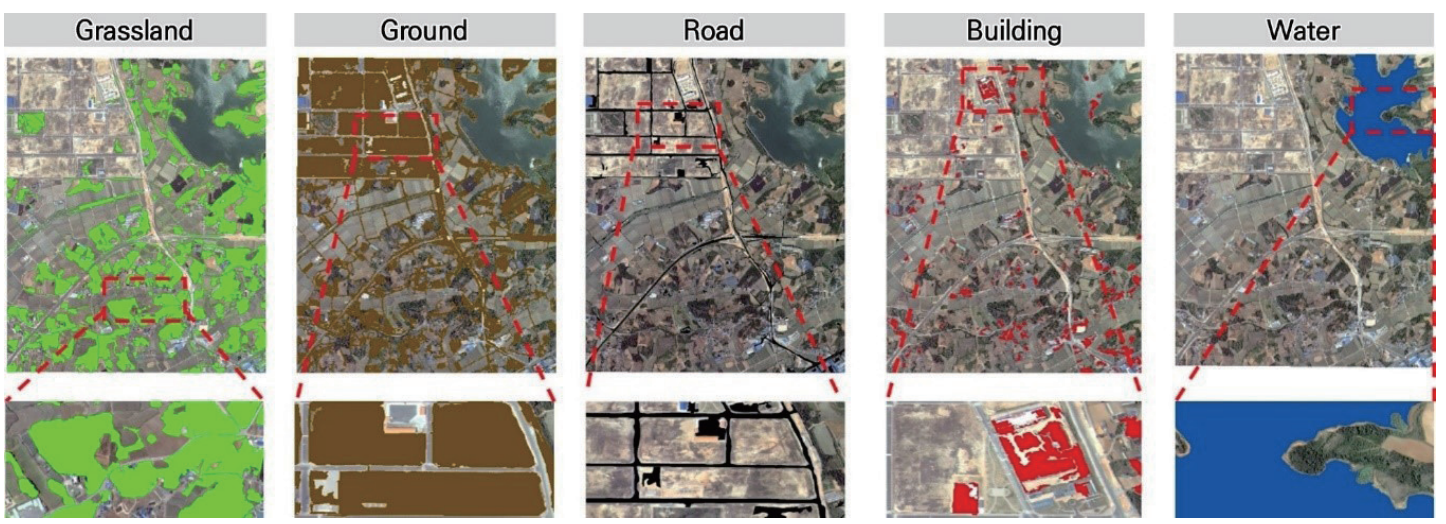

Fig. 15. (Color online) Results of geospatial feature extraction using the commercial SW (June 2015).
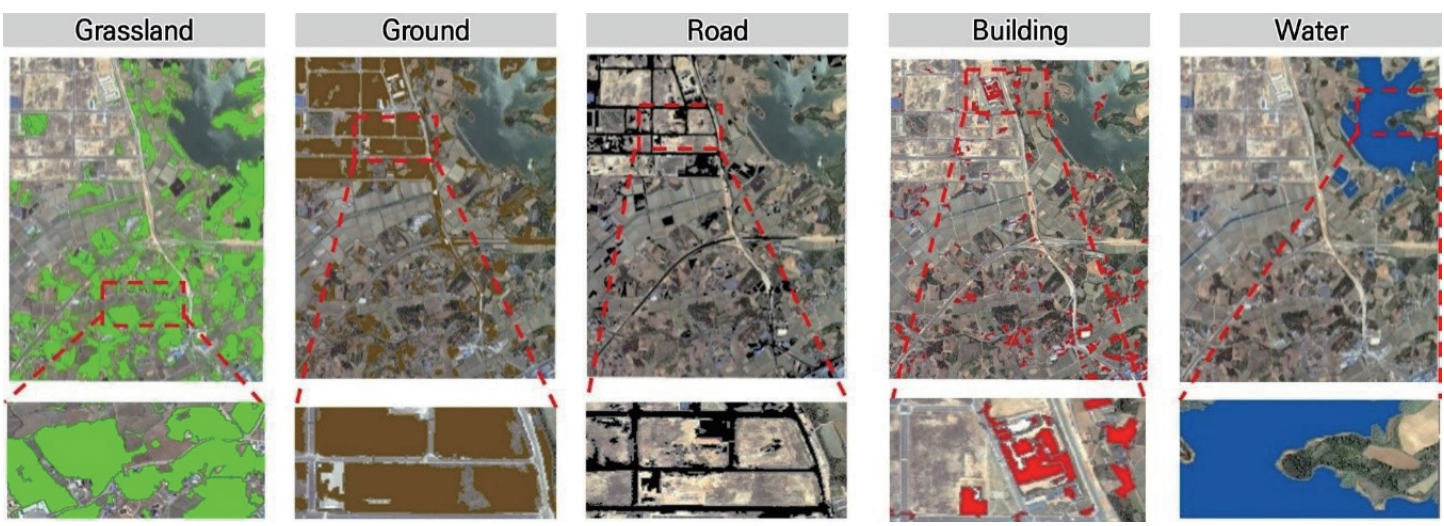

Fig. 16. (Color online) Results of geospatial feature extraction using the developed system (June 2015).

setting the same parameters and threshold values for both the commercial SW and the developed system. Figures 17 and 18, showing the geospatial features extracted using the commercial SW and the developed system, respectively, indicate the similar performances of both methods. 

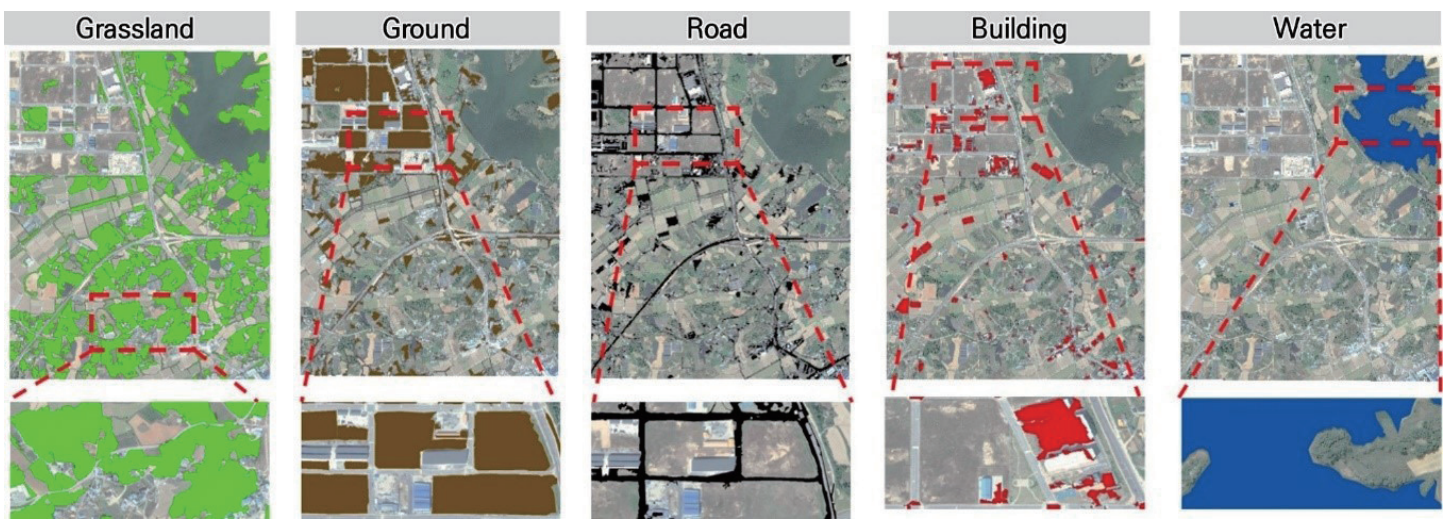

Fig. 17. (Color online) Results of geospatial feature extraction using the commercial SW (October 2017).
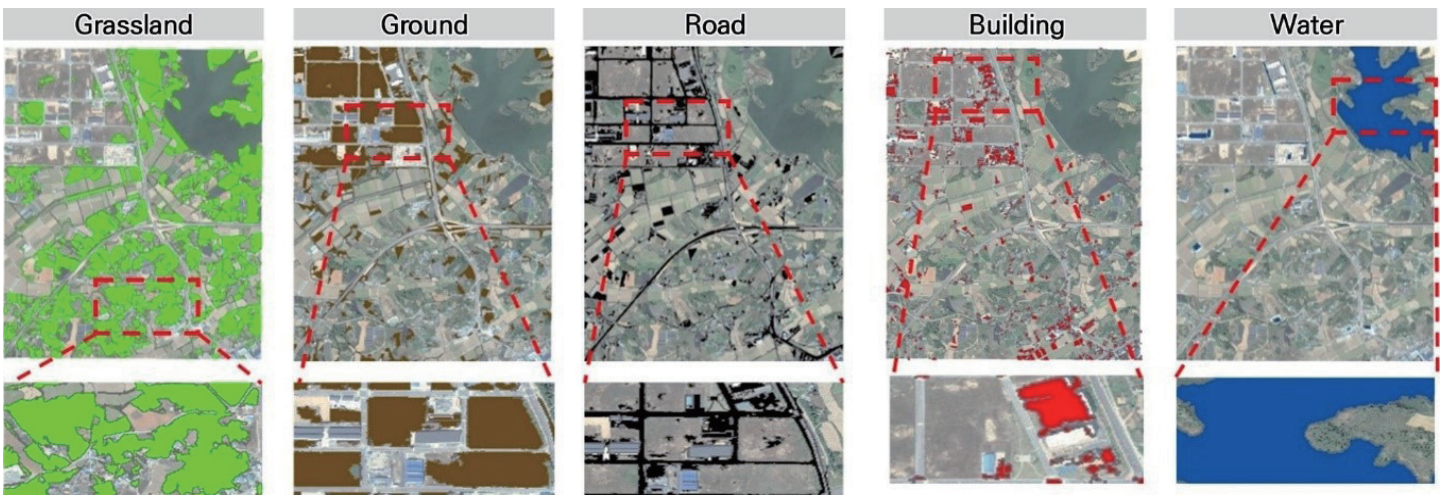

Fig. 18. (Color online) Results of geospatial feature extraction using the developed system (October 2017).

\subsection{Classification accuracy analysis of geospatial feature extraction results}

The classification accuracy of geospatial feature extraction results was analyzed using random points and an error matrix. The results showed that the geospatial features extracted by the commercial SW and the developed system indicate comparable performances, with classification accuracies higher than $90 \%$ for all items, as shown in Table 3. In other words, the goal of $90 \%$ or higher accuracy set in this study was satisfied.

Table 3

Results of classification accuracy analysis for geospatial features extracted using the commercial SW and the developed system.

\begin{tabular}{lcccc}
\hline \multirow{2}{*}{ Class } & \multicolumn{2}{c}{ Commercial SW } & \multicolumn{2}{c}{ Developed system } \\
\cline { 2 - 5 } & 2015.06 & 2017.10 & 2015.06 & 2017.10 \\
\hline Grassland & $98.00 \%$ & $92.20 \%$ & $97.60 \%$ & $91.80 \%$ \\
Ground & $96.40 \%$ & $95.00 \%$ & $96.20 \%$ & $94.80 \%$ \\
Roads & $93.80 \%$ & $91.20 \%$ & $93.80 \%$ & $91.20 \%$ \\
Buildings & $96.20 \%$ & $94.80 \%$ & $96.20 \%$ & $94.80 \%$ \\
Water & $99.40 \%$ & $99.20 \%$ & $99.40 \%$ & $99.20 \%$ \\
\hline
\end{tabular}




\section{Conclusions}

The aim of this study was to develop a geospatial feature extraction system for images from CAS500 satellites currently being developed by MOLIT, Republic of Korea. Using the system developed in an open-source environment, we investigated the possibility of the automatic extraction of geospatial features using KOMPSAT-3A satellite images, featuring similar specifications to CAS500 satellite images. Relative radiometric normalization was used for the automatic extraction of geospatial features. The same parameters and threshold values for image classification were set for the reference image and the relative radiometric normalized image in order to verify that geospatial features can be extracted. Furthermore, to develop a geospatial feature extraction system in an open-source environment, SAGA GIS was set as the development platform, spatial analysis libraries such as GDAL and PostgreSQL were adopted, and AVX2 and OpenMP were used to improve the image processing speed. The developed geospatial feature extraction system and commercial SW were used to extract geospatial features, and the classification accuracies were analyzed. The analysis results showed that the proposed system satisfies the goal of $90 \%$ or higher accuracy and demonstrates similar performances to the commercial SW. The system we developed is expected to be able to generate basic data that can be used to monitor forests, urban areas, and so forth.

A particular strength of the geospatial feature extraction technique proposed in this study is that it is implemented in an open-source environment to allow the automatic extraction of geospatial features item by item in conjunction with the relative radiometric normalization algorithm.

In the future, it is expected that a system capable of automatically extracting geospatial features can be developed if a regression equation that can automatically derive parameters and threshold values is developed and applied to the proposed geospatial feature extraction system. In addition, the automatic extraction of various geospatial features and the automatic production of thematic maps required for the work of MOLIT will be possible.

\section{Acknowledgments}

This research was supported by a grant (20SIUE-B148326-03) from the CAS 500-1/2 Image Acquisition and Utilization Technology Development Program, funded by the Ministry of Land, Infrastructure, and Transport of the Korean government, and by a National Research Foundation of Korea (NRF) grant funded by the Korean government (MSIT) (No. NRF2020R1A2C1102875). This research was also supported by Sangji University Research Fund (2019).

\section{References}

1 D. G. Lee, J. H. You, S. G. Park, S. H. Baeck, and H. J. Lee: Sens. Mater. 31 (2019) 10. https://doi.org/10.18494/ $\underline{\text { SAM.2019.2472 }}$

2 J. Pekel, A. Cottam, N. Gorelick, and A. S. Belward: Nature 540 (2016) 418. https://doi.org/10.1038/nature20584

3 A. M. Melesse, Q. Weng, P. S. Thenkabail, and G. B. Senay: Sensors 7 (2007) 12. https://doi.org/10.3390/ s7123209 
4 J. K. Lee, T. D. Acharya, and D. H. Lee: Sens. Mater. 30 (2018) 12. https://doi.org/10.18494/SAM.2018.1934

5 T. Whiteside and W. Ahmad: Proc. SSC2005 Spatial Intelligence, Innovation and Praxis: The National Biennial Conf. Spatial Sciences Institute (2005) 1225-1231.

6 H. J. Lee, J. H. Ru, and Y. G. Yu: J. Korean Soc. Geospat. Inf. Syst. 18 (2010) 73.

7 G. H. Mitri and I. Z. Gitas: Int. J. Appl. Earth Obs. Geoinf. 20 (2013) 60. https://doi.org/10.1016/j.jag.2011.09.001

8 A. Stumpf and N. Kerle: Remote Sens. Environ. 115 (2011) 2564. https://doi.org/10.1016/j.rse.2011.05.013

9 S. W. Myint, P. Gober, A. Brazel, S. Grossman-Clarke, and Q. Weng: Remote Sens. Environ. 115 (2011) 1145. https://doi.org/10.1016/j.rse.2010.12.017

10 D. Li, Y. Ke, H. Gong, and X. Li: Remote Sens. 7 (2015) 16917. https://doi.org/10.3390/rs71215861

11 F. Yuan and M. E. Bauer: Proc. ASPRS 2006: Annu. Conf. American Society for Photogrammetry and Remote Sensing (2006) 1667-51674.

12 D. G. Lee, S. R. Yang, and H. J. Lee: J. Korean Soc. Geospat. Inf. Sys. 28 (2020) 21. https://dx.doi.org/10.7319/ kogsis.2020.28.3.021

13 Y. J. Choung: J. Korean Assoc. Geogr. Inf. Stud. 18 (2015) 170. https://doi.org/10.11108/kagis.2015.18.1.170

14 D. Li, Y. Ke, H. Gong, and X. Li: Remote Sens. 7 (2015) 16917. https://doi.org/10.3390/rs71215861

15 M. Li, A. Stein, W. Bijker, and Q. Zhan: ISPRS 122 (2016) 192. https://doi.org/10.1016/j.isprsjprs.2016.10.007

16 D. H. Bui and L. Mucsi: Remote Sens. 13 (2021) 1700. https://doi.org/10.3390/rs13091700

17 H. G. Moon, S. M. Lee, and J. G. Cha: J. Korean Assoc. Geogr. Inf. Stud. 20 (2017) 1. https://doi.org/10.11108/ kagis.2017.20.1.001

18 Y. J. Choung: J. Korean Assoc. Geogr. Inf. Stud. 18 (2015) 135. https://doi.org/10.11108/kagis.2015.18.2.135

19 J. W. Choi: J. Korean Soc. Surv. Geodesy Photogramm. Cartogr. 33 (2015) 4. https://doi.org/10.7848/ ksgpc.2015.33.4.297

20 D. K. Seo, Y. H. Kim, Y. D. Eo, W. Y. Park, and H. C. Park: Remote Sens. 9 (2017) 11. https://doi.org/10.3390/ rs9111163

21 G. Hong and Y. Zhang: Int. J. Remote Sens. 29 (2008) 2. https://doi.org/10.1080/01431160601086019

22 Y. Du, P. M. Teillet, and J. Cihlar: Remote Sens. Environ. 82 (2002) 1. https://doi.org/10.1016/S00344257(02)00029v-9

23 H. Zhou, S. Liu, J. He, Q. Wen, L. Song, and Y. Ma: Int. J. Remote Sens. 37 (2016) 19. https://doi.org/10.1080/0 1431161.2016.1213922

24 O. A. D. Carvalho, R. F. Guimaraes, N. C. Silva, A. R. Gillespie, A. T. Comes, C. R Silva, and A. P. F. D Carvalho: Remote Sens. 5 (2013) 6. https://doi.org/10.3390/rs5062763

25 S. G. Biday and U. Bhosle: J. Comput. Sci. 6 (2010) 9. https://doi.org/10.3844/jcssp.2010.1027.1036

26 S. M. Ya'allah and M. R. Saradjian: Inf. Fusion 6 (2005) 3. https://doi.org/10.1016/j.inffus.2004.12.002

27 R. Hang, Q. Liu, H. Song, Y. Sun, F. Zhu, and H. Pei: IEEE J. Sel. Top. Appl. Earth Obs. Remote Sens. 10 (2017) 1. https://doi.org/10.1109/JSTARS.2016.2574802

28 Y. Bai, P. Tang, and C. Hu: Remote Sens. 10 (2018) 3. https://doi.org/10.3390/rs10030432

29 D. G. Lee, J. H. You, and H. J. Lee: J. Korea Soc. Geospat. Inf. Sci. 26 (2018) 3. https://doi.org/10.7319/ kogsis.2018.26.3.013

30 D. K. Seo and Y. K. Eo: J. Korean Soc. Surv. Geod. Photogramm. Cartogr. 36 (2018) 6. https://doi.org/10.7848/ ksgpc.2018.36.6.515

31 C. Y. Oh, S. Y. Park, H. S. Kim, Y. W. Lee, and C. U. Choi: J. Korean Assoc. Geograp. Inf. Stud. 13 (2010) 1. https://doi.org/10.11108/kagis.2010.13.1.089

32 H. J. Lee, J. H. Lu, and S. Y. Kim: J. Korean Soc. Geospat. Info. Syst. 19 (2011) 1.

\section{About the Authors}

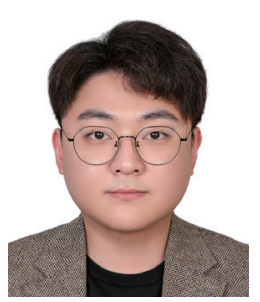

Dong Gook Lee received his B.S., M.S., and Ph.D. degrees from Sangji University, Republic of Korea, in 2015, 2017, and 2021, respectively. From 2018 until 2020, he lectured at Yeoju Institute of Technology. Since 2021, he has been working on digital twin research tasks at ALLforLAND Co., Ltd. His interests are in UAV, satellite image utilization, various types of GIS and RS analyses, and digital twins. (leedg1210@naver.com) 


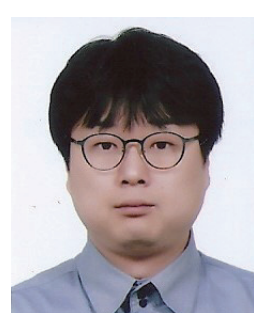

Ji Ho You received his B.S., M.S., and Ph.D. degrees from Sangji University, Republic of Korea, in 1996, 2006, and 2011, respectively. From 2006 until 2015, he lectured at Sangji University and Yeoju Institute of Technology. Since 2015, he has worked on GIS DB tasks at Chung-ang Aerosurvey Co., Ltd. His interests are in UAV, satellite image utilization, and building spatial information. (sjce96@daum.net)

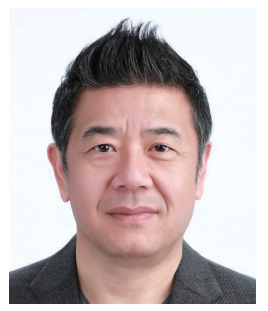

Hyun Jik Lee received his B.S. degree from Chungbuk National University, Republic of Korea, in 1984 and his M.S. and Ph.D. degrees from Yonsei University, Republic of Korea, in 1986 and 1992, respectively. Since 1996, he has been a professor at Sangji University, Republic of Korea. He has worked on numerous research studies and projects related to measurements and GIS. His research interests are in the utilization of various types of spatial information. (hjiklee@sangji.ac.kr) 